\title{
Mehr als nur eine Hautkrankheit
}

\author{
Das Krankheitsmanagement der Psoriasis ist eine multidisziplinäre Aufgabe.
}

Die Psoriasis oder Schuppenflechte beschränkt sich nicht auf die Haut - häufig sind auch andere Organe betroffen.

Der Leidensdruck von Psoriasis-Patienten ist hoch: Nicht nur Schmerzen und Jucken machen den Betroffenen zu schaffen, sondern auch die psychosozialen Folgen des „anders Aussehens“. „Erst vergangenen Freitag war eine 21-jährige Patientin bei mir, die sich seit Jahren nicht mehr ins Freibad getraut hat", sagt Prim. Dr. Werner Saxinger, Leiter der Abteilung für Haut- und Geschlechtskrankheiten am Klinikum Wels-Grieskirchen. In Österreich leiden zwei bis drei Prozent der Bevölkerung rund 250.000 Menschen - an der Schuppenflechte. „Leider sind viele Patienten unbzw. unterbehandelt", erklärt Saxinger. „Oft dauert es mehrere Jahre, bis sie einen Dermatologen aufsuchen und adäquat behandelt werden."

\section{Keine Heilung aber Linderung}

Psoriasis ist eine chronische Erkrankung, was die Behandlung schwierig macht. Es wird davon ausgegangen, dass es sich um eine autoimmune T-Zell-mediierte Immunreaktion handelt, bei der das Immunsystem körpereigenes Gewebe als fremd ansieht und angreift - in den betroffenen Geweben entsteht ein proinflammatorisches Milieu. Eine Heilung ist nicht möglich, allerdings kann durch moderne Behandlungsmethoden eine Linderung der Symptome bewirkt werden. Dabei spielen Biologika eine maßgebliche Rolle. Sie greifen zielgerichtet ins Entzündungsgeschehen ein und blockieren ganz spezifisch Abläufe des Immunsystems, die für die chronische Entzündung verantwortlich sind

$\mathrm{Zu}$ allem Überfluss ist Psoriasis nicht notwendigerweise auf die Haut beschränkt. „Bis zu 48 Prozent der Patienten haben auch Entzündungen der Gelenke.

\section{Systemische Erkrankung}

Psoriasis-Patienten haben auch häufiger Diabetes, Störungen des Fettstoffwechsels, Herz-Kreislauf-Erkrankungen, Depressionen sowie Morbus Crohn und Colitis ulzerosa“, erläutert OA Priv. Doz. Dr. Paul Sator, Leiter der Psoriasis Ambulanz der Abteilung Dermatologie des KH-Hietzing. „Die Diagnostik und Therapie der Begleiterkrankungen erfordert eine enge interdisziplinäre Zusammenarbeit zwischen Dermatologen, Rheumatologen, Allgemeinmedizinern, Internisten und Augenärzten. Eine Früherkennung kann nur möglich sein, wenn das Zusammenspiel der verschiedenen Fächer gut funktioniert."

Quelle: Pressekonferenz anlässlich des Welt-Psoriasis-Tages, am 16. Oktober in Wien

\section{Hier steht eine Anzeige.}

\section{Springer}

